\title{
La investigación narrativa de saber pedagógico: una perspectiva sociocultural*
}

\author{
DIEGO MAURICIO BARRERA QUIROGA**
}

Recepción: 15 de octubre de 2019

Aprobación: 27 de diciembre de 2019

Forma de citar este artículo: Barrera, D. (2020). La investigación narrativa de saber pedagógico: una perspectiva sociocultural. Cuadernos de Lingüística Hispánica, (35), 199-220.

10.19053/0121053X.n35.2020.10238

* Artículo de revisión.

** Magíster en Educación, licenciado en Educación Básica con énfasis en Humanidades y Lengua Castellana, y abogado, Universidad de la Amazonia. Correo: barreradiego1990@gmail.com @ https://orcid.org/0000-0002-8880-3443 


\section{Resumen:}

Esta investigación reconstruyó narrativamente las experiencias de una docente en la Universidad de la Amazonia, a través del proceso de historización y rehistorización de sus prácticas pedagógicas. Nos apoyamos en la investigación cualitativa, acompañada del método narrativo, así como en un trabajo dialógico con la participante en todos los momentos de la investigación. El enfoque sociocultural permitió orientar la visión de la informante en el proceso. A su vez, la modalidad investigativa posibilitó indagar sobre la formación, el desarrollo profesional y la acción docente. También, el análisis de la trayectoria docente logró develar conocimientos provenientes de los acontecimientos vividos en el ejercicio pedagógico, descifrar los aprendizajes construidos con el tiempo y la experiencia como síntesis que emergió a través de los relatos. Finalmente, la comprensión narrativa contribuyó en la construcción de sentido en el mundo y a identificar las instancias específicas que marcaron la sedimentación del saber pedagógico.

Palabras clave: investigación narrativa; reconstrucción narrativa; saber pedagógico; experiencia (actividad social); enfoque sociocultural.

\section{Narrative Research of Pedagogical Knowledge: A Sociocultural Perspective}

\section{Abstract}

This research narratively rebuilt the experiences of a professor at the University of the Amazon, through the process of historizing and re-historizing her pedagogical practices. We rely on qualitative research and narrative method, as well as on dialogical work with the participant at all moments of the investigation. The sociocultural approach allowed guiding the informant's vision in the process. In turn, the investigative modality made it possible to inquire about training, professional development and teaching action. Also, analysis of teaching career uncovered knowledge from the events lived in the pedagogical exercise, decipher the learning built over time and the experience as a synthesis that emerged through the stories. Finally, narrative understanding contributed to the construction of meaning in the world and to identify specific instances that marked the sedimentation of pedagogical knowledge.

Keywords: narrative research; narrative rebuilding; pedagogical knowledge; experience (social activity); sociocultural approach. 


\section{La recherche narrative du savoir pédagogique: une perspective socioculturelle}

\section{Résumé:}

Ce travail de recherche a reconstruit narrativement les expériences d'une enseignante de l'Université d'Amazonie à travers le processus d'historisation et de réhistorisation de ses pratiques pédagogiques. Pour atteindre l'objectif proposé, nous nous appuyons sur une recherche qualitative, accompagnée de la méthode narrative, ainsi que sur un travail dialogique avec la participante à tous les moments de la recherche. L'approche socioculturelle a permis de diriger le regard de l'enseignante citée (cf-supra) dans le processus. À son tour, la méthode de recherche a permis de s'interroger au sujet de trois volets : la formation, l'essor professionnel et la pratique pédagogique. Aussi, l'analyse de la formation de l'enseignante a permis, tout d'abord, de dévoiler les connaissances des événements vécus dans la pratique; de déchiffrer, ensuite, l'apprentissage construit au fil du temps et, finalement, de présenter par le biais de récits l'expérience comme une synthèse émergente. Enfin, la compréhension narrative a contribué à la construction du sens dans le monde et à l'identification des cas spécifiques qui ont marqué le triage des savoirs pédagogiques.

Mots clés: recherche; narrative; reconstruction narrative; savoirs pédagógique; vécu (activité sociale); aproche socio-culturel.

\section{A investigação narrativa do conhecimento pedagógico: uma perspectiva sociocultural}

\section{Resumo}

Este trabalho de pesquisa reconstruiu narrativamente as experiências de uma professora da Universidade da Amazônia através do processo de historização e rehistorização de suas práticas pedagógicas. Para atingir o objetivo estabelecido, contamos com uma pesquisa qualitativa, acompanhada pelo método narrativo, além de um trabalho dialógico com o participante em todos os momentos da pesquisa. A abordagem sociocultural permitiu orientar 0 olhar do informante no processo. Por sua vez, a modalidade investigativa possibilitou indagar sobre treinamento, desenvolvimento profissional e ação docente. Além disso, a análise da carreira docente conseguiu descobrir o conhecimento dos eventos vivenciados no exercício pedagógico, decifrar o aprendizado construído ao longo do tempo e a experiência como uma síntese emergente das histórias. Por fim, a compreensão narrativa contribuiu para a construção de sentido no mundo e para identificar os casos específicos que marcaram a sedimentação do conhecimento pedagógico

Palavras-chave: pesquisa narrativa; reconstrução narrativa; conhecimento pedagógico; experiência (atividade social); abordagem sociocultural. 


\section{Introducción}

En este trabajo se exploraron las narrativas de las experiencias de una profesora del Programa de Lengua Castellana y Literatura, en la Universidad de la Amazonia. El centro de atención en el proceso de construcción y reconstrucción narrativa de sus experiencias es la identificación del saber pedagógico presente en sus relatos.

No obstante, el objetivo de identificar saberes pedagógicos en las narrativas construidas y reconstruidas por los participantes sostiene un propósito más amplio: el de la reflexión producida en el proceso de investigación y su incidencia sobre las prácticas docentes, las prácticas investigativas, las prácticas de proyección social y las prácticas de autoformación en el Programa de Lengua Castellana y Literatura. De acuerdo con González y Caro (2018), el papel de la reflexión se debe tomar como un principio que ayuda a mejorar la práctica docente y la actividad profesional. Además, permite reconocer errores e involucra posibles soluciones.

La investigación está basada en un enfoque sociocultural centrado en la reconstrucción narrativa de la experiencia, diálogo polémico que constituye un proceso de construcción de significados. Así, al tiempo que se conversa sobre las experiencias, se escuchan las propias voces y las voces que se incorporan a las propias, surgen nuevos interrogantes, reflexionamos sobre nuevas alternativas, jugamos en los bordes del sentido común y nos aventuramos por los umbrales de nuevas formas de significar (Gergen, 2009, p. 5).

La investigación planteó varias situaciones desde una perspectiva crítica. La primera buscó precisar que la formación y el ejercicio de la actividad pedagógica en lengua y literatura corresponden al entendido de las ciencias sociales y humanas, y que, para ser pertinentes con sus fenómenos, tendrían que indagarse desde puntos de partida ontológicos, epistemológicos, teóricos, metodológicos y axiológicos propios de las ciencias sociales. La segunda se propuso plantear un marco de referencia amplio desde el enfoque sociocultural de la investigación narrativa, para poner en coherencia la investigación en el programa, con el objeto de estudio y trabajo de los profesores del Programa de Lengua Castellana y Literatura de la Universidad de la Amazonia, desde los presupuestos antes mencionados en los que se sustenta la licenciatura. En la tercera situación se tuvo en cuenta que la investigación narrativa permite organizar el trabajo de indagación desde lo disciplinario, lo multidisciplinario y lo interdisciplinario. Lo disciplinario, en cada uno de los componentes del programa y de la formación docente; lo multidisciplinario, en el sentido en que posibilita la integración del trabajo de investigación con investigadores 
de diferentes disciplinas del programa y de otros programas y facultades en torno a un proyecto; e interdisciplinario, en cuanto que el mismo objeto de estudio - las narrativas pedagógicas - se constituye en fenómeno de estudio desde varias disciplinas. Y la cuarta pretendió reconocer la investigación desde las concepciones propias de las ciencias sociales y las humanidades, ya que permite organizar la formación de los profesores de lengua y literatura con un grado mayor de unidad y coherencia formativa en el campo educativo.

\section{Marco teórico}

En este trabajo entendimos la actividad investigativa como un proceso de acción e interacción significativa y comunicativa entre los participantes, de tal manera que se reconoce que en el acto de investigar existe una relación de influencia recíproca entre los sujetos que actúan e interactúan y que la construcción de efectos de sentido es intersubjetiva, porque el sujeto enunciador no puede manejar lo que quiere decir (y lo que dice sin querer) sin la consideración de su interlocutor y de las formaciones discursivas en las que se desenvuelven (convergencia discursiva).

La investigación narrativa, en el marco del enfoque sociocultural, cuyas bases fueron propuestas por Vygotski (1995; 2009) y su grupo (Luria, 1980; Leontiev, 1977), se trabajó para fortalecer, examinar e interpretar la actividad humana en relación con el contexto histórico, social y cultural en el que tuvo lugar esta investigación.

Es necesario aclarar que el concepto de narrativa es utilizado frecuentemente como sinónimo de historia o relato (life histories of life stories) (Goodson, 2001; Hatch \& Wisniewski, 1995; Bisquerra, 2004), lo que hace necesario identificar determinados elementos que componen la narrativa y su cercana construcción con la humanidad. Así, por ejemplo, Todorov (1973) propone dos conceptos: historia y relato. Por historia se comprende el curso cronológico de hechos; en cambio, el relato es el resultado del orden inmerso en una historia; un relato compone la historia o la historia compone varios relatos y fragmenta las "grandes narrativas". Asimismo, Ducrot y Todorov (1983) ubican el relato como "un texto referencial con temporalidad representada" (p. 340).

Ahora bien, el conocimiento narrativo se refiere a lo humano y sus ejemplos más conocidos son la historia y la literatura. Generalmente se asume como saber que está construido a partir de la experiencia y que es significado a través de relatos. Los métodos utilizados en este tipo de conocimiento son de carácter interpretativo, narrativo o hermenéutico. No buscan la verdad, sino la verosimilitud; es decir, que se parezcan a la experiencia del mundo de la vida. Los discursos con los que se significa este tipo 
de conocimiento son de base narrativa y se refieren a intenciones, deseos e historias particulares. Este tipo de conocimiento reconoce las voces de los participantes y la del investigador como voces en diálogo. La narrativa, esencialmente, construye y da sentido a las experiencias vividas y, en última instancia, del tránsito vivido de los seres humanos y de los demás a través del mundo (Moen, 2006).

Se ha optado por la reconstrucción del saber pedagógico de los profesores de lengua mediante narrativas, porque "la narrativa es el esquema primario mediante el cual la existencia humana se transforma en significación" (Polkinghorne, 1988, p. 1). Todos los seres humanos a lo largo de la vida hemos vivido experiencias e interacciones comunicativas con los otros, con nosotros y con el mundo y, cuando damos cuenta a los otros de esas experiencias, las organizamos como narrativas; en consecuencia, los seres humanos organizamos las experiencias vividas en formas narrativas. Sin embargo, no solamente narrativizamos lo vivido, también relatamos las experiencias de otras personas.

Esto conduce a decir que "[el] saber es experiencia ancestral o experiencia sedimentada en el curso de una vida" (Zambrano, citado por Domingo \& De Lara Ferré, 2010, p. 51), una reflexión del maestro que contribuye en la elaboración de su saber pedagógico, desde una práctica cotidiana dirigida a la transformación, evaluación y cambios, propios de la mediación dialógica. Ahora bien, toda transformación intelectual es una reestructuración parcial o total de la organización de saberes, desde la cual cada individuo formula, lleva a cabo o hace real sus interrelaciones consigo mismo, con la naturaleza, con la sociedad y con los saberes que en ella se disponen. Es un acto creativo (Gallego-Badillo, 1992) que se construye a través de la interacción curricular e investigación en el aula (Stenhouse, 1998). También, una acción reflexiva y contextuada (Schön, 1983; 1987) que vincula los valores, las interpretaciones y la visión de los docentes (Gudmundsdottir, 1998).

Según Philpott (2014), la investigación narrativa se ha venido abriendo campo en la educación, particularmente en la definición del papel de las narrativas en la formación inicial de los profesores, el papel de las narrativas en la conformación de la identidad de los profesores y el rol que tienen las narrativas en la estructuración del conocimiento de los docentes.

En Iberoamérica fueron de interés, tanto para fortalecer la comprensión de la investigación (biográfica) narrativa, como para ver sus aplicaciones al campo de la educación, los trabajos de Bolívar (2002), Bolívar y Domingo (2006), Larrosa (1995), Da Cunha (1997), Suárez, Ochoa y Dávila (2003), el Grupo de Investigaciones en Educación 
y Estudios Culturales, Universidad Nacional de Mar del Plata y Ministerio de Educación Ciencia y Tecnología de Argentina (MECTI) (2007a, 2007b).

En ese orden de ideas, también podemos reseñar los trabajos de Meneguzzi (2004), quien investiga sobre la formación de los docentes en la universidad desde la formación pedagógica y la calidad en el desempeño profesoral, y Díaz (2004, 2006), quien indaga sobre la construcción del saber pedagógico en los docentes universitarios.

En Colombia las tendencias del movimiento pedagógico se pueden incluir en una especie de resistencia a las imposiciones del "currículo a prueba de maestros" con los que se ha tratado de educar a los sectores populares de la población colombiana. Así, por ejemplo, la Expedición Pedagógica Nacional (2001) buscó recuperar la perspectiva de la educación desde los maestros como sujetos de la pedagogía. Desde la metáfora del viaje se ve la pedagogía como una práctica contextuada en un momento de la historia, la sociedad y la cultura. En ese marco se hicieron esfuerzos de investigación que recientemente han producido resultados, como el trabajo de Parra y Castañeda (2014), quienes hacen una radiografía de la inclusión precaria de los docentes; el de Vasco (1997), quien desarrolló una investigación donde involucra maestros, estudiantes y diferentes niveles de saberes educativos desde el saber pedagógico, y el de Tamayo (2004), el cual optó por un estudio en la formación pedagógica del docente universitario en Colombia, identificando la necesidad de nuevas estrategias didácticas y su mejoramiento para los procesos de enseñanza en la educación superior.

En el plano regional, los trabajos de Gabriel Murillo $(2008,2010,2015)$ son un ejemplo de como se ha venido desarrollando el interés sobre la investigación (biográfica) narrativa en las prácticas pedagógicas en la zona del país donde se piensa con mayor seriedad la educación y la pedagogía. Asimismo, la investigación de Yarza, Ramírez, Franco y Vásquez (2015) y la divulgación de varios trabajos que dan cuenta de "la experiencia pedagógica, la práctica pedagógica y el saber pedagógico" (Martínez, 2015, p. 9), recogidos en el libro Práctica y experiencia. Claves del saber pedagógico docente, de Martínez (2015).

\section{Metodología}

A partir de la comprensión de que los métodos propios de las ciencias naturales no son los más eficaces para realizar la búsqueda del conocimiento en los fenómenos sociales, se ha venido incrementando el uso de métodos narrativos, como puede evidenciarse en la inclusión de capítulos sobre el método narrativo en publicaciones dedicadas a difundir el desarrollo de los métodos en las ciencias sociales. En ese sentido, pueden confrontarse 
Creswell (2014), De Fina y Georgakopoulou (2015), Clandinin (2012), Andrews, Day Sclater, Squire y Tamboukou (2004), y Hühn, Meister, Pier, Schmid y Schönert (2009).

Por eso, la ruta metodológica propuesta en el trabajo inició con los elementos sugeridos por la investigación cualitativa, porque integra formas alternas para aproximarse al mundo social. Girar hacia interpretaciones particulares 0 situadas y vencer las generalidades, los universales y las estructuras permiten acentuar al sujeto en acción.

Para los investigadores de la Universidad del Zulia, Rodríguez, Vera y Vargas (2011), la investigación cualitativa

[...] es un campo de saberes científico-humanístico con diversas raíces históricas y construcciones onto-epistemológicas, que progresivamente ha construido un corpus teórico propio y denso, [por eso] se hace necesario una mirada reposada para aprehender en forma apropiada las profundidades de tal corpus teórico y sus potencialidades para la generación de teorías y nuevos saberes en el mundo de las ciencias humanas. (p. 28).

Esta opción metodológica comprende una realidad en el abanico extenso para entender y conocer el mundo humano, pero se diferencia de otras por la posibilidad alterna ante la "certeza" cuantitativa. Ante el choque entre enfoques cuantitativos y cualitativos, algunos han querido reducir su discusión ontológica, epistemológica y axiológica a la utilización de números en el primer caso y el no uso en el segundo. Sin embargo, la diferencia principal se ve en la intención y el tipo de mundo que cada enfoque percibe.

Por otro lado, el objetivo de la investigación presenta la construcción como un componente esencial en el entender; es decir, no hablamos de recolección, sino que nos acercamos al performance, a la actuación, a la conversación y las contingencias. También, se considera que el dato no se encuentra dado o presente por fuera de la relación entre el "informante" y el investigador, por el contrario, se propone ver las herramientas de obtención informativa como elecciones de acuerdo al momento específico. Para el caso en concreto, utilizamos notas, entrevistas, interacciones orales, narrativas discontinuas, documentos escritos, fotografías, etc. (Biglia \& Bonet-Martí, 2009; Sanz, 2005; Connelly \& Clandinin, 1995). Además, la investigación narrativa se inscribió en la secuencia de eventos y datos diacrónicos; descripción de hechos, efectos de los hechos y procedimientos interpretativos. Polkinghorne (1995) dirá "[...] creación de una síntesis [más] que una categorización". Uso de los datos hacia la emergencia de una trama temática.

Asimismo, partimos de un enfoque sociocultural en la investigación narrativa y del reconocimiento de que los profesores son muy importantes en el desarrollo 
de la pedagogía y del currículo, por lo tanto, hay que prestar especial atención a sus experiencias individuales y grupales, escuchar sus propias voces y las narrativas acerca de su trabajo y de su vida. Pero iqué hicimos como investigadores? Tomando en cuenta lo anterior, nos interesamos por la complejidad que trae la interacción con docentes, porque la comprensión de lo individual no es posible sin una consideración simultánea del contexto institucional, el contexto del sistema educativo, su currículo, las tendencias pedagógicas, la formación discursiva sobre lo educativo y lo pedagógico, y el contexto social y cultural (el discurso de la profesora en concierto con otros discursos que circulan en la sociedad sobre los temas que ella trata), por lo cual, hay que responder a esta exigencia de la investigación en términos de habilitar a quienes realizan el trabajo de articulación de los niveles personales, microsocial, mesosocial y macrosocial. Es decir, el construir y reconstruir las experiencias en forma narrativa abre a los participantes a la reflexión y al cambio sobre lo que son y lo que hacen, permite entrelazar las voces singulares en una trama de relatos compartidos, por tanto, hacer un esfuerzo por reinventar el presente y proyectar acciones hacia algún futuro. Por eso, la investigación se apoyó en el método narrativo, porque las conexiones o entrecruzamiento de los relatos ayudaron en el proceso de comprensión y posicionamiento de lo sujetos como profesionales políticos que no conciben la práctica pedagógica sin una visión del mundo. También, encontramos que esta perspectiva de conocimiento considera "los procesos de subjetivación que conducen a valorar al otro como sujeto con capacidad de acción y reflexión con historia, con un proceso de devenir en tanto sujeto relacional" (De los Ríos, Ramírez, Mejía \& Zapata, 2015, p. xix).

En conclusión, para el análisis de la información obtenida se utilizó la técnica de "tringulación secuencial" (Bolívar, Fernández \& Molina, 2005) en coherencia con la historización y rehistorización (Creswell, 2014), aplicadas sucesivamente a las narrativas. Esta técnica posibilitó identificar las tramas narrativas del conjunto histórico conseguido. Y a partir de esto, mostrar las relaciones del sujeto con los diversos espacios vividos: familia, formación (escolar y profesional), institucionalidad, práctica pedagógica, construcción de saber y perspectiva de la lengua, así como su enseñanza.

\section{Resultados}

El lenguaje como herramienta mediadora de la significación está cercano a la reflexión, gracias a la pluralidad de voces que se encuentran en un discurso. Somos capaces de relatar, fragmentar los momentos, guardar determinados acontecimientos de nuestra vida, relacionar los espacios y la temporalidad al narrar. Además, se es consciente 
del carácter dialógico en la investigación narrativa, hecho fundamental para los procesos investigativos en educación.

Por eso, con la narración de la profesora Guillermina -participante- se pudieron descifrar los aprendizajes construidos con el tiempo y la experiencia como síntesis emergente a través de los relatos. El ser humano se construye con sus historias, experiencias y aprendizajes que interactúan entre los saberes prácticos, teóricos y disciplinarios.

Yo soy la última de ocho bijos; la mayoría de los bijos fueron mujeres, lo que jugó un papel fundamental en la formación personal, debido a la relación solidaria entre las mujeres que se fortalece en las diferentes etapas de la vida. Esa relación, que surgió en la niñez, ha sido la constante de mi vida, hasta boy, somos unas hermanas: las mejores hermanas.

La compañía de su familia, especialmente de sus hermanas, señala la importancia de la formación inicial y del acompañamiento a los primeros pasos.

Con los hermanos se desarrollaron unas dinámicas diferentes a las nuestras. Fueron dos hombres mayores. Con ellos no tenemos mayores relaciones. Somos parte de la búsqueda que hicieron mis padres en el Caquetá, abriendo vida y territorio, creando condiciones en la región.

La familia va a ser el centro de desarrollo personal, a pesar de la ruptura con sus hermanos. La idea patriarcal de la familia se resiente por la mayoría femenina en la que se ha apoyado la crianza, generación tras generación.

Mi papá fue un maestro de obra que trabajó por muchas zonas. Primero, en la comisaría y, luego, en la intendencia. Le asignaban trabajos de construcción de puestos de salud, puentes, escuelas, etc. Eso me dio la oportunidad de vivir en varios lugares del departamento: San José del Fragua, Belén de los Andaquies, Solano, Guacamayas, Paujil. Mi papá iba a hacer los trabajos y como yo era la menor de todas, me llevaba. Mi mamá no me dejaba, siempre andaba con ellos.

La figura de los padres es un referente importante en la construcción familiar. La protección y compañía apoyan el desarrollo de quien inicia su vida junto a los suyos. Viajar

1 Ubicado en el municipio de San Vicente del Caguán, Caquetá. 
abre oportunidades de descubrir y conocer, así como la idea de libertad. Acompañaban al padre y desde allí se configura la cohesión familiar, ya que se gira en torno a la posibilidad productiva del patriarca. Van detrás del proveedor, aprendiendo de la vida y con la vida, dentro de las prácticas sociales a las que él está articulado.

La narrativa facilita un marco de referencia como creador y transmisor de conocimiento. El sujeto activa su saber a través de la reconstrucción narrativa, ya que ciertas prácticas conscientes no se mantienen eternas, sino que se movilizan y demandan de la reconstrucción para lograr claridad en la persona. En la reconstrucción, los resultados no prueban nada, solo inclinan, ya que la búsqueda de ciertas explicaciones 0 concepciones implican otras respuestas e ideas.

Guillermina será el reflejo de las experiencias vividas. Las condiciones sociales y culturales comprenden el material de formación del sujeto. La reconstrucción permite ir identificando las experiencias como referente que da significado e identidad.

\section{Eso me marcó de por vida porque yo me crie en un ambiente de much a libertad, no solamente la libertad que me daba poder conocer las cosas, vivirlas, sino la libertad que ellos me dieron porque yo no vivía constreñida ni vigilada todo el tiempo, ya que ellos tenían sus ocupaciones.}

La libertad será la herencia infantil y familiar, y por la que estará constantemente exigiendo en su entorno. Descubrir, indagar o explorar a través de la experiencia, desde la vivencia. El contacto con la naturaleza será importante para la vida, para el desarrollo de la futura mujer.

Con esto se demuestra el reconocimiento de sí mismo, ese "Yo" determinado en la narración que muestra su experiencia sin desconocer la incidencia de otros. Mirar los acontecimientos desde un sentido crítico y reflexivo permite tejer el significado y darle a su experiencia fuerza valorativa, ya que reconoce el resultado histórico que la envuelve.

Explorar, conocer e indagar por la vida enlaza las condiciones básicas para el conocimiento inicial.

La casa de nosotros era grande y al fondo tenía una cocina de leña: abi se hacían los alimentos. A mi hermana mayor no le gustaba que doña María cocinara, entonces, ella le decía: - "Doña María, yo hago el almuerzo y usted cuénteles historias a mis hermanas". Y, doña María se sentaba en una silla, abría el libro y se ponía a contarnos, a leernos Las mily una noches. Lo que uno no entendía, ella lo mostraba e ilustraba con el libro: me imagino que ella se inventaría cosas, haría los acomodos, pero nos entretenía. Claro, mi hermana 
era para que no molestáramos, nos mantenía cautivas durante mucho tiempo. Ella nos contaba historias, bistorias de terror y nos leía este libro. El libro era bermosísimo, todo ilustrado, con hojitas finas, parecidas a las bojitas con las que antes se bacian las biblias y unas ilustraciones bermosísimas: un libro muy lindo. Ella nos mostraba las ilustraciones y nos leía los cuentos, nos tenía boras hasta que terminábamos e íbamos a comer.

El tránsito entre el aprendizaje por cuenta propia y el aprendizaje vinculado a la enseñanza, como se estila en la escuela, tendrá sus incidencias en la vida educativa y social; la primera, sujetada al descubrir, escudriñar, investigar y leer desde las condiciones de su entorno; la segunda, guiada por repertorios establecidos y adheridos a actividades individuales, asignando lecturas a sus participantes. Tenemos, entonces, una lectura del mundo y sus fenómenos de forma natural (leer el texto del mundo) y el nuevo tipo de lectura (leer en el mundo del texto) como hacía María para ellas. Eso significa, por un lado, el ejercicio de indagación con su contexto y, por otro, la condición guiada desde las herramientas establecidas por la convencionalidad social. La libertad del recuerdo se muestra con espontaneidad. Además, las narraciones acompañadas de la imagen permiten ilustrar la historia al compás de la espera por la comida. La casa grande y el fogón de leña constituyen un espacio de encuentro y diálogo. Buen ejemplo de ellos son las comunidades primitivas que se caracterizaron por transmitir sus saberes alrededor del fuego, enseñaban sus experiencias de caza a los menores, quienes atentos escuchaban la palabra de sus mayores. En el círculo está la palabra, los abuelos se encargan de rodear la llama del saber avivando el calor tradicional, alimentando el espíritu para mediar la naturaleza y la comunidad material.

Las descripciones en el proceso narrativo muestran que la actividad humana es una manifestación del mundo guiada por propósitos, situaciones y secuencias presentadas en la sociedad, que, para el caso concreto, corresponde a cada hecho vivido como grano de arena en la playa de la vida. Los libros seguirán marcando la búsqueda; su lectura, formando el sueño profesional y el encuentro con la realidad se conjugará para determinar el devenir de la vida. Sus hermanas seguirán jugando un papel esencial en la formación de la futura formadora. Alimentar el conocimiento y el cuerpo comprende una visión conjunta, cuidar del cuerpo y nutrir el juicio para tomar consciencia de su entorno.

Seguir los pasos de Guillermina no es otra cosa que "la sedimentación de lo vivido: encontrar las huellas de lo que ha pasado; seguir el rastro de quien ya transitó o está transitando, descifrarla, concertarla” (Ferraroti, 1990, p. 110). El relato me lleva a descubrir que desde la experiencia y con cada ensayo o error va puliendo su ejercicio docente. Al narrar encontramos las condiciones del contexto, siguiendo los pasos que 
influenciaron, los lazos afectivos y comunicativos, las dinámicas generacionales y las construcciones que en colectivo se van consolidando. Volver sobre lo vivido genera una proyección hacia el futuro y una reflexión del ejercicio presente. No hay actividad sin reflexión y reflexión sin nuevas proyecciones educativas.

Ya en la universidad me dediqué a la parte que tiene que ver con profesionalización y bubo una ligera variación en los intereses, aunque me sigue gustando la literatura. Por esas cosas de la vida yo terminé trabajando más la parte de la lengua y me fui enamorando de ella, encarretando con ella, porque me di cuenta de que la lengua es un elemento fundamental de lo que nosotros somos, hacemos, sentimos; entonces, casi todo mi trabajo profesional estuvo y a muy dirigido hacia la parte de la formación lingüistica y la formación especifica de la didáctica de la lengua. La misma formación que recibi en la Universidad Nacional me orientó hacia allá, hacia el conocimiento de la lengua, no solamente la lengua nuestra: la materna, sino de otros sistemas lingüísticos, entonces, uno al hacer el trabajo de comparación entre los diferentes sistemas se da cuenta de que nosotros tenemos en nuestro lenguaje un instrumento maravilloso, nosotros no seríamos lo que somos sin eso y eso le da una valoración muy grande al trabajo que debe hacer con la lengua. Es extraño que nosotros todo el día utilizamos la lengua y baya gente que no se interese por saber ¿qué es?, ¿cómo funciona?, ¿para qué sirve? Y que hagamos un uso tan acrítico de ella, tan irreflexivo, para mí es un cuestionamiento que hago a los estudiantes, es como si la gente utilizara todo el tiempo una cosa y no se detuviera un momento a pensar: bueno y esta cosa cómo es, como si la cotidianidad trivializara la importancia que la lengua tiene en nuestro diario vivir y en nuestro diario profesional, entonces, yo me metí más por esa línea, por la línea de la lengua. Además, porque el trabajo con las comunidades indigenas le abre a uno las perspectivas, ellos tienen una visión muy diferente de lo que es la lengua, lo que es la importancia de la lengua en esos grupos, en la forma como los cohesiona en un grupo particular con una identidad propia que es un poco diferente de lo que nosotros vivimos aquí en la cultura occidental.

En la narración, las experiencias se concentran en una secuencia modulada por la trama, reconstruida a través del relato. Este proceso de construcción y reconstrucción diferencia una narrativa a la lista de hechos cronológicamente engranados. Será, entonces, el proceso narrativo el que signifique, suministre y conecte al ser humano con las condiciones sociales y culturales, además, entienda las contingencias particulares del sujeto sin llegar a las generalidades o verdades inamovibles. 
Por otro lado, es importante resaltar del relato la mirada al código lingüístico dentro de los estudios del lenguaje y su asimilación de lenguaje a lengua en olvido a las otras formas culturales de significar y comunicar. Esto es relevante dentro del saber pedagógico por la idea de lengua/lenguaje que se maneja, los objetivos de la enseñanza de la lengua, los contenidos y los métodos con los que se enseñan ciertas formas de entender y explicar la capacidad humana para semiotizar.

Otro elemento de análisis es la enseñanza de la lengua que corresponde al campo de formación adquirido y que reflexiona desde una perspectiva alimentada por la experiencia. Es importante destacar algunas ideas que se tejen a través del relato. La mirada del mundo lingüístico, el estudio de las destrezas comunicativas y las teorías en torno a la enseñanza corresponden al análisis dispuesto en el diálogo libre y crítico.

Por otro lado, la reflexión en el relato adquiere importancia al dotarlo de sentido por las voces que se van reconociendo en el camino, por las historias enriquecidas desde la acción vivida. Los relatos serán testimonio de la práctica pedagógica que humaniza y contribuye a la construcción de identidad. Ser capaz de asignar significado al contexto afianza la identidad profesoral, articula la visión docente como creador y no reproductor de datos, temporaliza las circunstancias de la época, sitúa al sujeto y su discurso, y ayuda a resolver problemáticas locales y regionales porque se identifica con la comunidad. Cada vez que se construye y reconstruye un relato, los narradores evalúan las experiencias recordadas como importantes 0 no, y ligan los momentos para descifrar el significado particular en el individuo. Por tanto, las narrativas irradian con la memoria, las reflexiones pedagógicas y la reconstrucción del saber pedagógico que el docente ha sedimentado; en este caso particular, con la profesora Guillermina:

Yo enseño la lengua para todo. La lengua hay que enseñarla para aprender a pensar y organizar el pensamiento. Hay que enseñarla porque nos permite funcionar en una sociedad donde, mayoritariamente, somos alfabetas. Hay que enseñarla porque todas las prácticas que nosotros desarrollamos cotidianamente tienen que ver con la lengua; bay que enseñarla porque nosotros como profesores enseñamos a través de los usos de la lengua; hay que enseñarla porque la función cognitiva se desarrolla con el lenguaje: ¿cómo me acerco a los objetos del conocimiento? Pues, a través del lenguaje. Cada una de las actividades que uno desarrolla está abi y, por lo tanto, abi está su importancia, su razón de ser, abí está el valor.

Nuevamente, reconoce la importancia del lenguaje como mediador del conocimiento y del desarrollo social. El soporte semiótico, especialmente el lenguaje, 
jugará un papel fundamental porque está en función del proceso cognitivo, transformador y reflexivo del ser humano. Enseñarlo, entonces, cobra su importancia por la incidencia social y cultural entre los sujetos. El aula y la interacción con los estudiantes serán el espacio para extender la función crítica y de conocimiento que se gesta gracias al lenguaje. El lenguaje y su construcción narrativa aportan en la edificación de la sociedad, primero, por medio del acto comunicativo y, segundo, desde la cohesión social.

\section{Discusión}

Debemos mencionar que el propósito de esta investigación no fue solamente interpretar los sentidos que le imponen los actores a los saberes pedagógicos, sino que, en los procesos de reconstrucción narrativa de esos saberes, se produjeron transformaciones de las identidades y las prácticas docentes en una dirección favorable a los intereses de la sociedad, de los grupos y de los individuos. Demos una idea: la práctica educativa profesoral se entiende como la construcción e interpretación de las situaciones escolares producto de los resultados de las decisiones adoptadas, las reflexiones de los acontecimientos vividos y la proyección significativa de la experiencia transitada. A saber: hablar de construcción social, experiencia y reflexión nos conduce al conocimiento profesional del profesor como un cúmulo de hechos que envuelve las actividades escolares y sociales.

En último lugar, las dimensiones sociales, políticas, económicas y culturales en la educación también contribuyen a la comprensión de las actividades escolares: ir más allá del instrumento y edificar espacios interactivos que ayuden en la reflexión consciente del momento áulico. Identificar el contexto social tendrá, entonces, posibilidades de desarrollo valorativas y aportes en la concepción democrática de ciudadanía. Un proyecto curricular, en este sentido, desenmascara prejuicios separatistas entre la comunidad, alienta la inclusión y planifica la defensa de principios deliberativos, eliminando las restricciones o represiones cultivadas por el desconocimiento del medio, estimula la reflexión crítica y propicia condiciones para la reconstrucción del tejido social.

\section{Conclusiones}

El valor narrativo en el estudio de la enseñanza se explica con la idea de McEwan (1998), quien manifiesta que "[al] contar historias acerca de la docencia [...] aprenderemos a enseñar mejor" (p. 25), también, a comprender el mundo desde la exploración, y la búsqueda de múltiples maneras para entender la enseñanza.

Para Elbaz (citada por Mendieta, 2013), la historia: 
[...] is the very stuff of teaching, the landscape within which we live as teachers and researchers, and within which the work of teachers can be seen as making sense. This is not merely a claim about the aesthetic or emotional sense of the notion of the story with our intuitive understanding of teaching, but an epistemological claim that teachers' knowledge in its own terms is ordered by story and can best be understood in this way². (p. 146).

La investigación narrativa no involucra exclusivamente al individuo como sujeto escindido, sino que también se refiere al contexto, a la inclusión del profesor en un sistema escolar, curricular, ideológico, pedagógico y político que transita por procesos transformadores.

Cualquier docente que contemple estas dimensiones sabe que el aula contiene numerosas situaciones e imprevistos por resolver no solamente desde la formación teórica, sino,0020por el contrario, requiere de condiciones que el contexto produce, ya sea intencionado o no, pero sí distintivo por los sujetos que interactúan. En otras palabas, las tensiones por solucionar no se limitan a lo disciplinar, estas mantienen propiedades emanadas de la relación y la cotidianidad, de la contingencia y diversidad, de la intervención que en el marco del aula se identifica y aviva la práctica, de la simultaneidad y la imprevisibilidad. Las situaciones confusas e indeterminadas manifiestan en la práctica hechos de aula que contribuyen al conocimiento profesional. Los aspectos problémicos ayudan a la reflexión en y sobre la acción. Cuando atendemos estos problemas, seleccionamos y consideramos las situaciones, los límites y programamos nuestra atención para modificar 0 aportar en la superación de las dificultades. Esto significa que nos enmarcamos en un proceso interactivo, resultado, en su mayoría, de acciones y reacciones contextuales. De plano se puede afirmar que reducir lo práctico a "quienes aplican" y lo teórico a "quienes conocen", separa la integralidad del conocimiento y el mundo. Compartir las experiencias derivadas de las prácticas posibilita eliminar la concepción jerárquica a través de la realidad educativa. Ni una teoría como razón única y sí una práctica contextuada. Para los profesores el concepto de teoría produce temor. Primero, porque se cubre bajo el manto de expertos que legitiman una práctica educativa determinada, haciéndolos extraños. Segundo, porque el conocimiento se ubica

2 “...] es la materia misma de la enseñanza, el paisaje dentro del cual vivimos como profesores e investigadores, y dentro del cual se puede ver que el trabajo de los docentes tiene sentido. Esto no es simplemente una afirmación sobre el sentido estético o emocional de la noción de la historia con nuestra comprensión intuitiva de la enseñanza, sino una afirmación epistemológica de que el conocimiento de los maestros en sus propios términos está ordenado por la historia y se puede entender mejor de esta manera". (Traducción libre). 
en las generalidades de las prácticas, dejando la sensación de incapacidad de construir y definir elementos pedagógicos por parte del profesor. Y tercero, este temor crece con las definiciones de los investigadores que predeterminan las prácticas educativas sin ubicar la realidad concreta del entorno escolar. No hay confianza ante las definiciones que parecen "verdades" y forzosas de admitir. Se sienten lejanos por las generalidades cuando ellos viven la "práctica imperfecta" y oculta que escapa de su control. En consecuencia, los teóricos pasan a ser recibidos desde una perspectiva tecnócrata que toma decisiones provistas de presupuestos positivistas sin considerar las capacidades que trae el conocimiento práctico de los profesores en el ejercicio educativo. A esto se le adiciona el papel legitimador de los gobiernos y su aplicación desde las políticas educativas inconsultas, que generan poca confiabilidad. Ahora, no se trata de rechazar a ultranza las posiciones teóricas, porque estas sirven de contraste con el conocimiento práctico, incluso pueden contribuir ante los saberes rutinarios e inertes. La urgencia está en estrechar lazos para mejorar la calidad de la enseñanza y nutrir los aprendizajes desde lo colectivo, pasar del temor a la confianza, del rechazo a la aceptación y de la jerarquía al diálogo colaborativo.

Ningún sujeto está aislado, por el contrario, las narrativas de su contexto permean la relación individual generando, para el caso docente, diversos niveles de sentir y entendimiento con la experiencia. La relación social, cultural, política y económica de su entorno constituirá en el docente una percepción particular, transformando sus prácticas escolares y mejorando la responsabilidad social del ejercicio pedagógico. Para Riessman (2008), con la investigación narrativa "los relatos son artefactos sociales que nos hablan tanto de una sociedad y una cultura como lo hacen de una persona o un grupo" (p. 105).

Abreviemos: el aporte de la investigación biofráfica-narrativa en la reconstrucción del "saber pedagógico" y "saber social"3 (Baracaldo, 2007, p. 21) se entiende como conocimiento de los saberes específicos, en las disímiles culturas. También es encuentro, diálogo intersubjetivo en donde los agentes educativos desarrollan procesos de enseñanzaaprendizaje o, como lo puntualiza Zuluaga (1979), en el marco del saber pedagógico.

Hoy podemos asegurar que el trabajo del docente-investigador, en cualquier disciplina, está relacionado con la palabra escrita y la composición (narrativa) oral. La historia en el campo de la educación, por poner un ejemplo, se alimenta de las narraciones, experiencias, acontecimientos, vivencias y reconstrucciones de la realidad que posteriormente se verán formadas en la producción de textos, artículos o investigaciones,

3 Dos conceptos emergentes en Colombia, a partir del trabajo de la práctica pedagógica y del movimiento pedagógico. 
pero también en sus relatos. Por eso, la investigación narrativa se presenta como una nueva forma de indagación, en el sistema educativo, que adquiere interés en los cuerpos de estudio y sistematización de las experiencias.

Según Clandinin (1985), la falta de reconocimiento y éxito del profesor está vinculada a una visión que minimiza su conocimiento, negando su papel activo y autónomo sin conocimientos prácticos. Por esta razón, la investigación narrativa pretende ofrecer a los investigadores la oportunidad de mostrar la complejidad de la enseñanza y el aprendizaje al público (y a los profesores). Las narraciones, asimismo, permiten que las voces de los profesores y estudiantes sean escuchadas a la par, ampliando las percepciones a través de las intervenciones polifónicas que alimentan la comprensión educativa, las motivaciones, las luchas, las pérdidas, así como las ideologías que guían sus caminos de aprendizaje mutuo.

Por lo tanto, al recoger las narrativas de Guillermina, encontramos que la familia, la tutoría de sus hermanas, fueron el aporte esencial en la proyección profesional. Las experiencias vividas contribuyeron a la determinación futura de su ejercicio pedagógico. Ya la formación disciplinada, fuera de la Universidad de la Amazonia, afianzó lo que se había moldeado desde el ámbito familiar para luego ponerlo al servicio de la institución que la vio nacer intelectualmente. En cada narración se mostró siempre contraria al establecimiento, a las condiciones de la época, decidida a aprender, sin olvidar que el aprendizaje es un proceso inacabado.

En consecuencia, las narraciones inevitablemente permean al maestro y su conocimiento, generando comprensión del trabajo y el aprendizaje en contexto. El docente se muestra y construye por medio de la interacción (diálogo). Revela su significado y articula conocimiento pedagógico desde la experiencia. Proceso activo desarrollado por el docente que implica relaciones sociales constantes. Igualmente, las narrativas y su construcción de conocimiento se concentran en identificar patrones significativos del sujeto a través de los relatos, relacionando el contexto social y cultural con los entornos de aprendizaje (trabajo) del docente, permitiendo la comprensión de las condiciones involucradas.

En últimas, para los docentes el desarrollo pedagógico lo constituyen elementos de representación que se adquieren en la experiencia y sus acontecimientos, en el deber del vivir y actuar de los maestros, y su contexto educativo. 


\section{Referencias}

Andrews, M., Day, S., Squire, C. \& Tamboukou, M. (2004). Narrative Research. In C. Seale, G. Gobo, J. Gubrium \& D. Silverman (eds.), Qualitative Research Practice (pp. 97-112). London: Sage.

Baracaldo, M. (2007). Investigación de los saberes pedagógicos. Recuperado de https://www. mineducacion.gov.co/1621/articles-345504_anexo_13.pdf

Biglia, B. \& Bonet-Martí, J. (2009). La construcción de narrativas como método de investigación psicosocial. Prácticas de escritura compartida. Forum, 10(1), 1-25.

Bisquerra, R. (2004). Metodología de la investigación educativa. Madrid: La Muralla.

Bolívar, A. (2002). ¿De nobis ipsis silemus?: epistemología de la investigación biográficonarrativa en educación. Revista Electrónica de Investigación Educativa, 4(1), 41-62.

Bolívar, A. \& Domingo, J. (2006). La investigación biográfica y narrativa en Iberoamérica: campos de desarrollo y estado actual. Forum: Qualitative Social Research, 7(4), 1-43.

Bolívar, A., Fernández, M. \& Molina, E. (2005). Investigar la identidad profesional del profesorado: una triangulación secuencial. Forum: Qualitative Social Research, 6 (1), 12. Recuperado de https://www.researchgate.net/profile/Antonio_Bolivar/publication/277054166 Researching_Teachers'_Professional_Identity_A_A_Sequential_Triangulation/ links/5588522b08ae347f9bda96ff/Researching-Teachers-Professional-Identity-ASequential-Triangulation.pdf

Connelly, M. \& Clandinin, J. (1995). Relatos de experiencia e investigación narrativa. En J. Larrosa (ed.), Déjame que te cuente (pp.11-59). Barcelona: Laertes.

Creswell, J. W. (2014). Research Design: Qualitative, Quantitative, and Mixed Method Approaches (4th ed.). London: Sage.

Clandinin, D. J. (1985). Personal Practical Knowledge: A Study of Teachers' Classroom Images. Curriculum Inquiry, 15(4), 361-385. Doi:10.1080/03626784.1985.11075976

Clandinin, D. J. (ed.). (2012). Handbook of Narrative Inquiry: Mapping a Methodology. London: Sage.

Da Cunha, M. I. (1997). Conta-me agora!: as narrativas como alternativas pedagógicas na pesquisa e no ensino. Revista da Faculdade de Educação, 23(1-2).

De Fina, A. \& Georgakopoulou, A. (2015). The Handbook of Narrative Analysis. Hoboken, NJ, USA: Wiley- Blackwell. 
De los Ríos, V. A. Y., Ramírez, M. R., Mejía, L. M. F. \& Zapata, N. C. V. (2015). Narrativas de la educación especial en Medellín: una reconstrucción polifónica, 1966-2004. Medellín, Colombia: Universidad de Antioquia.

Díaz, V. (2004). Teoría emergente en la construcción del saber pedagógico. Telos, 6(2), 169-193.

Díaz, V. (2006). Construcción del saber pedagógico. Caracas: Fondo para el Fomento y Desarrollo de la investigación, Universidad Pedagógica Experimental Libertador.

Domingo, J. C. \& De Lara Ferré, N. P. (2010). Investigar la experiencia educativa. Madrid: Morata.

Ducrot, 0. \& Todorov, T. (1983). Diccionario enciclopédico de las ciencias del lenguaje. Madrid: Siglo XXI.

Expedición Pedagógica Nacional. (2001). Preparando el equipaje. Bogotá: Universidad Pedagógica Nacional.

Ferraroti, F. (1990). La historia y lo cotidiano. Barcelona: Península.

Gallego-Badillo, R. (1992). Saber pedagógico: una visión alternativa. Bogotá: Cooperativa Editorial Magisterio.

Gergen, K. J. (2009). An Invitation to Social Construction. London: Sage.

Goodson, I. (2001). The Story of Life History: Origins of the Life History Method in Sociology. Identity: An International Journal of Theory and Research, 1(2), 129-142.

Gudmundsdottir, S. (1998). La naturaleza narrativa del saber pedagógico sobre los contenidos. En H. McEwan \& E. Heran. (comps). La narrativa en la enseñanza, el aprendizaje y la investigación (pp. 52-71). Buenos Aires: Amorrortu.

Hatch, J. A. \& Wisniewski, R. (Eds.). (1995). Life History and Narrative. London: The Falmer Press.

Hühn, P., Meister, J. C., Pier, J., Schmid, W. \& Schönert, J. (2009). The Living Handbook of Narratology. Hamburg: Hamburg University Press.

Larrosa, J. (1995). Déjame que te cuente: ensayos sobre narrativa y educación. Barcelona: Laertes.

Leontiev, A. N. (1977). Actividad y conciencia. Moscú: Progreso.

Luria, A. R. (1980). Pensamiento y lenguaje. Barcela: Tontanella.

Martínez, R. M. P. (2015). Práctica y experiencia. Claves del saber pedagógico docente. Bogotá: Universidad de La Salle. 
McEwan, H. (1998). Las narrativas en el estudio de la docencia. En H. McEwan \& K. Egan (comp.), La narrativa en la enseñanza, el aprendizaje y la investigación (236-259). Buenos Aires: Amorrortu.

Mendieta, J. A. (2013). Narrative Research: An Alternative Approach to Study Language Teaching and Learning. Folios, (37), 135-147. Retrieved from http://www.scielo.org.co/scielo. php?pid $=$ S0123-48702013000100009\&script $=$ sci_arttext\&tlng $=$ en

Meneguzzi, A. (2004). Formación de profesores en la universidad: formación pedagógica y calidad en el desempeño profesional. En VIII Congreso de Educación a Distancia CREAD MERCOSUR/SUL Córdoba-Argentina. Recuperado de http://biblo.una.edu.ve/docu.7/ bases/anali/texto/Meneguzzi.pdf

Ministerio de Educación, Ciencia y Tecnología de Argentina. (2007a). ¿Cómo editar pedagógicamente los relatos de experiencias? Buenos Aires: Fundación Laboratorio de Políticas Públicas. Colección de Materiales Pedagógicos.

Ministerio de Educación, Ciencia y Tecnología de Argentina. (2007b). ¿Qué es la documentación narrativa de experiencias pedagógicas? Buenos Aires: Fundación Laboratorio de Políticas Públicas. Colección de Materiales Pedagógicos.

Moen, T. (2006). Reflections on the Narrative Research Approach. International Journal of Qualitative Methods, 5(4), 56-69. Retrieved from http://journals.sagepub.com/doi/ abs/10.1177/160940690600500405.

Murillo, G. J. (Ed.) (2008). Maestros contadores de historias. Medellín: Artes y Letras.

Murillo, G. J. (Ed.). (2010). Los maestros contadores de historias: relato de una experiencia de formación y escritura. En R. Lazzari, L. Barbosa \& M. Appezzato Pinazza (orgs.), Modos de narrar a vida: cinema, fotografía, literatura e educacâo (pp. 69-85). Sâo Paulo: Cultura Académica Editorial.

Murillo, G. J. (2015). (Coord.). Narrativas de experiencia en educación y pedagogía de la memoria. Buenos Aires: Editorial de la Facultad de Filosofía y Letras.

Parra, R. \& Castañeda, E. (2014). La vida de los maestros colombianos: interculturalidad y ciudadanía en la escuela. Bogotá: Convenio Andrés Bello, Universidad de Ibagué, Universidad Externado de Colombia, 4 vols.

Polkinghorne, D. E. (1988). Narrative Knowing and Human Sciences. New York: State University of New York Press.

Polkinghorne, D. E. (1995). Narrative Configuration in Qualitative Analysis. International Journal of QualitativeStudies in Education, 8(1),5-23.http://dx.doi.org/10.1080/0951839950080103 
Philpott, C. (2013). How is Teacher Knowledge Shaped by the Professional Knowledge Context? Minding our Metaphors. Journal of Curriculum Studies, 45(4), 462-480. D0I: $10.1080 / 00220272.2013 .796527$

Riessman, C. K. (2008). Narrative Methods for the Human Sciences. London: Sage.

Rodríguez, V. G., Vera, G. \& Vargas, A. M. (2011). Etnografía: una mirada desde corpus teórico de la investigación cualitativa. Omnia, 17(2),26-39. Recuperado de https://www.redalyc.org/ pdf/737/73719138003.pdf

Sanz, A. (2005). El método biográfico en investigación social: potencialidades y limitaciones de las fuentes orales y los documentos personales. Asclepio, 57(1), 99-115.

Schön, D. (1983). El profesional reflexivo, como piensan los profesionales cuando actúan. Barcelona: Paidós.

Schön, D. (1987). La formación de profesionales reflexivos. Hacia un nuevo diseño de la enseñanza y el aprendizaje de las profesiones. Barcelona: Paidós

Stenhouse, L. (1998). La investigación como base de la enseñanza. Madrid: Morata.

Suárez, D., Ochoa, L., \& Dávila, P. (2003). Narrativa docente, prácticas escolares y reconstrucción de la memoria pedagógica. Módulo I y II. Argentina: Organización de los Estados Americanos Agencia Interamericana para la Cooperación y el Desarrollo (AICD).

Tamayo, V. A. (2004). La formación pedagógica del docente universitario. Pedagogía y Saberes, (21), 29-35.

Todorov, T. (1973). Gramática del Decamerón. Madrid: Taller de Ediciones Josefina Detancor.

Vasco, E. (1997). El enseñar y la enseñanza. En H. Ospina \& L. López (comps.), Pedagogías constructivistas, pedagogías activas y desarrollo humano (pp. 303-314). Bogotá: Cooperativa Editorial Magisterio.

Vygotski, L. S. (1995). Pensamiento y lenguaje. Buenos Aires: Fausto. Recuperado de http:// abacoenred.com/wp-content/uploads/2015/10/Pensamiento-y-Lenguaje-Vigotsky-Lev.pdf

Vygotski, L. S. (2009). El desarrollo de los procesos psicológicos superiores. Barcelona: Crítica.

Zuluaga, O. L. (1979). Por una historia de las prácticas pedagógicas en Colombia. En Ponencia presentada en el II Congreso de Historia de Colombia. (1995). Pensamiento y lenguaje. Buenos Aires: Ediciones Fausto. Recuperado de http:// abacoenred.com/wp-content/uploads/2015/10/Pensamiento-y-Lenguaje-Vigotsky-Lev.pdf 